\title{
Review on: Detection of Diabetic Retinopathy using SVM and MDA
}

\author{
Shveta \\ Mtech Student, CEC Landran,PTU
}

\author{
Gurmeen Kaur \\ Assistant Professor \\ CEC, Landran,Punjab Technical University
}

\begin{abstract}
Diabetes is the commonest cause of blindness in the working age group these days. Diabetes can affect the sight of a patient and thus it results in causing glaucoma, cataracts and its most severe effect is on blood vessels inside the eye as the blood vessels are damaged and it reaches a condition known as "diabetic retinopathy" which can also be called as eye blindness due to diabetes. Automatic detection of retinal abnormalities is commonly performed for haemorrhages, micro aneurysms, cotton wool spot and hard exudates. However, if more attention is paid to it, there is worse case of retinal abnormality called neovascularisation but much research was not done to detect it. In this case, new blood vessels branch out due to extensive lack of oxygen in the retinal capillaries. So the automated analysis of human eye fundus image is an important task as it can later lead to sectional blindness or thorough blindness. If desired quota of measures is taken and methods are put into consideration then it can be potentially reduced to $50 \%$.In this paper, we present a review for the detection of DR using fundus images and approaches SVM and MDA.
\end{abstract}

\section{Keywords}

Diabetes, Diabetic Retinopathy, Neovascularisation, Fundus Image, SVM, MDA.

\section{INTRODUCTION}

In today's world, blindness is an emerging problem almost everywhere. Efforts are being put into action by the researchers to overcome this disease. Thus early detection of this condition is of utmost importance as it can help to stop the progress of this disease and thus keeping sight and preventing blindness [2]. Diabetes is a disease which occurs when the pancreas does not secrete enough insulin or the body of a person is not able to process it as before. The circulatory system is affected by this disease and it also includes retina. As diabetes keeps on progressing, the sight of diabetic patient may start to deteriorate and lead to diabetic retinopathy. About $10 \%$ of all diabetic patients have diabetic retinopathy, which is the primary cause of blindness. Since this type of blindness can be prevented if treatment can be started at an earlier stage. Thus, World Health Organization (WHO) advises yearly scanning of patients.

DR is a critical eye disease which can be regarded as manifestation of diabetes on the retina. The main stages of DR are non-proliferative diabetic retinopathy (NPDR) which is the earliest stage of diabetic retinopathy. In this condition, the damaged blood vessels in retina of eye starts leaking extra fluid and small amount of blood in the eye. Sometimes, deposits of cholesterol collected or some other fats from blood may leak into the retina. The second stage is proliferative retinopathy (PDR) which occurs mainly when a number of blood vessels in the retina close, which prevents enough blood flow. Thus it attempts to supply blood to that particular area where original vessels are closed [7].

As a result, new blood vessels grow in the retina. This is called revascularization. If retinal vessels are closed in large number, neovascularization can occur in the iris (the colored part of the eye). In this condition, the normal flow of the fluid is stopped or blocked by the new blood vessels. Due to this blockage, a pressure is build up in the eye, and in this particular condition, damage can be caused to the optic nerve. However, these new blood vessels are unusual and do not supply the retina with sufficient flow of blood. A scar tissue accompanies these new blood vessels that may also cause retina to detatch or wrinkle.

PDR is more dangerous and it may cause more severe loss of vision than NPDR because it can affect both the central and surrounding vision. DR is the leading ophthalmic pathological cause of blindness among people of working age in developed countries. The main cause of DR is abnormal increase in blood glucose level, which damages vessel endothelium, thus increasing vessel permeability. The first demonstration of DR is tiny capillary dilations known as micro aneurysms. As DR progresses, it causes neovascularization, macular edema, hemorrhages and in laterly retinal detachment.

Fundus imaging has significant role in diabetic monitoring since presence of retinal abnormalities are common. Diabetic retinopathy is asymptomatic. A fundus camera is used to capture images of the retina that are then read and graded by doctors. Fundus images are employed by ophthalmologists most of times for the detection and diagnosis of Diabetic retinopathy. An automated or computer-assisted analysis of diabetic patients' retina can help eye care specialist to screen large population of diabetic patients [1]

In the past years, so many researchers proposed many methods for the automatic analysis of features for analyzing diabetic retinopathy, which are useful for the treatment. But, these methods are only useful in classifying them subjectively. The proposed methods in all researches were not completely reliable and they were robust too as they did not provide any objective measurement of the features. They were mainly useful in analysis of the specific features on the retina, but do not provide a system as a whole for the automatic detection of different stages of DR. The Investigations done so far and algorithms developed are unable to detect early stage of retinopathy (NPDR) accurately. So here we present a new technique for the analysis of lesions using classifiers and the different metrics will be taken into consideration to achieve better results.

\section{A. Support Vector Machine (SVM)}

Support vector machine (SVM) is a supervised learning model with an associated learning algorithm that can analyse data and recognize patterns which are then used for regression analysis and classification. If we take a set of training examples, where each is marked as belonging to one of two defined categories, an SVM training algorithm will build up a model that will assign new examples to one or the other category, making it nonprobabilistic binary linear classifier. SVM model is the representation of examples defined as points in space that are mapped so that the examples of the different categories can be divided by a clear gap that is as large as possible. Fresh examples are then mapped into that same space and expected to belong to a category based on which side of the gap they fall on 
The Support Vector Machine (SVM) is a state-of-the-art classification method introduced in 1992 by Boser, Guyon, and Vapnik. The SVM classifier is widely used in bioinformatics (and other disciplines) due to its high accuracy. It is able to calculate and process the high-dimensional data such as gene expression and exibility in modelling diverse sources of data. A general category named as kernel methods, SVM belong to that category. This kernel method is an algorithm which depends on data specifically through dot-product. In this case, a kernel function can replace this dot product and it will compute the dot product in some high dimensional feature space possibly. It has two advantages: Firstly, it has the ability to generate non-linear decision boundaries using those methods which are designed for linear classifiers. Secondly, use of kernel functions will allow the user to apply classifier to the data that have no defined fixeddimensional vector space representation. The best suited example of such data in bioinformatics is sequence, either, protein or DNA or protein structure. Using SVMs effectively requires an understanding of how they work. When training an SVM the practitioner needs to make a number of decisions: that is how to preprocess data, what kernel should be used, and in the last, setting up the parameters of SVM and kernel. Uninformed choices may result in severely decreased performance. Our aim is to provide the user with a complete understanding of the choices and also to provide general usage guidelines. All the examples shown were generated using the PyML machine learning environment, which focuses on kernel methods and SVMs.

\section{1) Properties of SVM}

SVM posses following properties:

- Flexibility of choosing a similar function.

- $\quad$ Simplicity of solution when dealing with large data sets support vectors are specifically used to specify the separation.

- It has the ability to handle large feature spaces - complexity is not dependent on the dimensionality of the feature space.

- Overfitting can be controlled by soft margin.

- Nice math property: a simple convex optimization problem is guaranteed to converge to one global solution.

- Does feature Selection.

2) Applications of SVM

SVM is being used successfully in many real-world problems as a classifier.

- gait recognition

- $\quad$ text (and hypertext) categorization

- $\quad$ image classification

- bioinformatics (Protein and Cancer classification) character recognition

\section{B. Multilinear Discriminant Analysis (MDA)}

Here we present a novel approach to solve the supervised dimensionality reduction problem by encoding an image object as a general tensor of second or even higher order. MDA is a discriminant tensor paradigm, where numerous interconnected minor discriminative subspaces are derived. Then, a novel approach, called k-mode optimization, is conferred to reiteratively learn these subspaces by unfolding the tensor along different tensor specifications. This algorithm is known as Multilinear Discriminant Analysis (MDA), which has the following characteristics:
- Numerous interconnected subspaces can be thrown in together to discriminate different classes,

- For classification problems involving higher order tensors, the MDA algorithm can avoid the bane of dimensionality dilemma and alleviate the small sample size problem

- The calculating cost in the initial stage is reduced to a large extent owing to the reduced data dimensions in k-mode optimization.

MDA is general supervised dimensionality reduction framework. It can avoid the dimensionality dilemma because the latter is performed in a much lower-dimension feature space than the traditional vector-based methods like LDA do.

MDA also resolves the problem of small sample size as the size is effectively multiplied by a large scale.

MDA contains more feature dimensions as it is not theoretically limited by number of classes in data.

The cost of computation is reduced to a larger extent because mode optimization is performed on feature space of smaller size at each step.

MDA extensions from vector to tensor for feature extraction as well as data representation are general and they can also be applied in combination with SVM and many other algorithms to improve the effectiveness and algorithmic learnability.

Due to the above characteristics, MDA is expected to be a more general and suitable algorithm for pattern classification problems in image analysis where objects can be encoded in tensor representation. It is different from the traditional subspace learning criterion which derives only one subspace. In our approach numerous interconnected subspaces are obtained through the optimization of the criterion where the number of the subspaces is determined by the order of the feature tensor used.

\section{LITERATURE SURVEY}

In the previous years, there have been multitudinous studies for the detection of diabetic retinopathy using different techniques and features.

Sohini Roychowdhury, Dara D. Koozekanani and Keshab K.Parhi (IEEE, 2013): proposed a computer-aided screening system (DREAM) that evaluate fundus images with varying illumination and range of view, and introduce a severity grade for diabetic retinopathy (DR) using machine learning. Classifiers like k-nearest neighbor (kNN), Gaussian Mixture Model (GMM), support vector machine (SVM), and AdaBoost are analyzed for classifying retinopathy lesions from non-lesions. GMM and kNN classifiers are considered to be the best classifiers for bright and red lesion allocation, respectively.

Murugan.R, Dr.Reeba Korah, (IJAIT, 2012): proposed a new automated methodology to detect the optic disc (OD) in retinal images. It uses line operator which gives higher percentage of detection. Its purpose is to naturally detect the position of the optic disc in digital retinal fundus images taken by fundus camera. The process starts with changing the RGB image input into its LAB component. The image is then smoothed with the help of bilateral smoothing filter. After this, line operator is used for the purpose of filtering. And then gray orientation and binary map orientation is carried out and then with the use of the resulting maximum image variation, the area where OD is present is found.

V. Vijayakumari, N. Suriyanarayanan, (EJSR, 2012): proposed a method which shows that for detecting exudates, the 
blood vessel can be segmented first and then the lesions are detected. Moreover the thickness of the blood vessel can be calculated from which the severity of diabetic retinopathy can be calculated. Matching and then relating the methods, the morphological operators can be used for the detection of blood vessel which is the simplest approach.

(Berrichi Fatima Zohra , Benyettou Mohamed): proposed a computer based approach for detecting diabetic retinopathy stage using color fundus images. The features are eradicated from raw images using image processing method and then afterwards it is transferred to the support vector machine (SVM).

Asha Gowda Karegowda, Asfiya Nasiha, M.A.Jayaram, A.S .Manjunath (IJCA, 2011): proposed a method where back propagation neural network is used to detect exudates. In the process of evaluation, the data which is used for detecting diabetic retinopathy is publically available dataset DIARETDB1. The optic disk was eliminated to limit the optic disk from interfering with exudates detection. Significant features were identified from the images after preprocessing by using two methods: Decision tree and GA-CFS. These methods are used as input to the BPN model to detect the exudates and non-exudates at pixel level.

\section{PROPOSED WORK}

Design and develop a new technique for the analysis of lesions in the retinal image using SVM and MDA and also detection of neovascularization and vascular beading. The results will be correlated with the other existing techniques using different metrics like Specificity, Sensitivity, PSNR (Peak Signal to Noise Ratio), AUC (Area under ROC Curve), time and Accuracy.

- Specificity: It is sometimes called the true negative rate. It measures the proportion of negatives which are correctly identified as such (e.g., the percentage of healthy people who are correctly identified as not having the condition)

- Sensitivity: It is also called the true positive rate or the recall rate in some fields. It measures the proportion of actual positives which are correctly identified as such (e.g., the percentage of sick people who are correctly identified as having the condition),

- PSNR: It is an engineering term for the ratio between the maximum possible power of a signal and the power of corrupting noise that affects the fidelity of its illustration. Because many signals have a large dynamic range, PSNR is usually depicted in terms of the logarithmic decibel scale.

- $\quad$ AUC: Area under the curve (AUC) is the area under the curve (mathematically known as definite integral) in a plot of concentration of drug in blood plasma against time. Typically, the area is measured at the time when the drug is administered and ended when the concentration in plasma is negligible.

\section{ACKNOWLEDGMENT}

I would like to thank my guide, family and my friends who helped me to accomplish this.

\section{REFERENCES}

[1] Sohini Roychowdhury, Student Member, IEEE, Dara D. Koozekanani, Member, IEEE and Keshab K.Parhi Fellow, IEEE (2013)," DREAM: Diabetic Retinopathy Analysis using
[2] Machine Learning", IEEE.

[3] Doaa Youssef, Nahed H. Solouma, NILES, Cairo University, Giza, Egypt (2012), "Accurate detection of blood vessels improves the detection of exudates in color fundus images", ELSEVIER.

[4] Murugan.R, Dr.Reeba Korah, (2012)," An Automatic Screening Method To Detect Optic Disc In The Retina", International Journal of Advanced Information Technology (IJAIT) Vol. 2, No.4.

[5] LiliXu, ShuqianLuo, (2010), "A novel method for blood vessel detection from retinal images", BioMedical Engineering OnLine.

[6] Mohammed AlRawi, Munib Qutaishat, Mohammed Arrar, (2006), "An improved matched filter for blood vessel detection of digital retinal images", Computers in Biology and Medicine, pp $262-267$.

[7] Herbert F. Jelinek , Michael J. Cree , Jorge J. G., May (2007), "Automated segmentation of retinal blood vessels and identification of proliferative Leandro , João V. B. Soars and Roberto M. Cesar, Jr. A. Luckie diabetic retinopathy “, Optical society of America, 24, pp 14481456.

[8] Priya.R , Aruna.P, (2011), "Review of automated diagnosis of diabetic retinopathy using the support vector machine", IJAER.

[9] Edgardo FelipeRiveron1 and Noel Garcia Guimeras, (2006), "Extraction of Blood Vessels in Ophthalmic Color Images of Human Retinas", CIARP 2006, LNCS 4225, pp. 118 126, Springer Verlag Berlin Heidelberg.

[10] Bevilacqua V., Cambò, S.Cariello, L.Mastronardi , G ., (2005), "A combined method to detect Retinal Fundus Features", Conference on EACDA, Italy.

[11] V. Vijayakumari, N. Suriyanarayanan, (2012)," Survey on the Detection Methods of Blood Vessel in Retinal Images", European Journal of Scientific ResearchISSN 1450-216X Vol.68 No.1 (2012), pp. 83-92@ EuroJournals Publishing, Inc.

2012

http://www.europeanjournalofscientificresearch.com

[12] Shilpa Joshi, Dr P.T. Karule, (2012),"Retinal Blood Vessel Segmentation", International Journal of Engineering and Innovative Technology (IJEIT) Volume 1, Issue 3.

[13] Berrichi Fatima Zohra, Benyettou Mohamed,"Automated diagnosis of retinal images using the Support Vector Machine (SVM)", 1Laboratoire de Modélisation et Optimisation des Systèmes Industriels: LAMOSI. Faculté des Sciences, Département d'Informatique, USTO. B.P. 1505 EL M'NAOUER 31000 ORAN - ALGERIE.

[14] Asha Gowda Karegowda, Asfiya Nasiha, M.A.Jayaram, A.S .Manjunath, (2011), "Exudates Detection in Retinal Images using Back Propagation Neural Network", International Journal of Computer Applications (0975 8887) Volume 25- No.3. 